\title{
Comunidades de prática: uma revisão bibliográfica sistemática sobre casos de aplicação organizacional
}

\section{Communities of Practice: a systematic literature review about practical cases in organizations}

\author{
Flávia Roberta Fernandes ${ }^{1}$, Tiago Alves Cardoso ${ }^{1}$, Lisiane Zynger Capaverde ${ }^{1}$, Helena de Fátima Nunes Silva ${ }^{1}$ \\ ${ }^{1}$ Universidade Federal do Paraná - UFPR, Curitiba, PR, Brasil
}

Autor para correspondência/Mail to: Flávia Roberta Fernandes (flaroberta@gmail.com)

Recebido/Submitted: 11 Maio 2016; Aceito/Approved: 28 Jun. 2016

\begin{abstract}
Copyright (c) 2016 Fernandes et al.. Todo o conteúdo da Revista (incluindo-se instruções, política editorial e modelos) está sob uma licença Creative Commons Atribuição-NãoComercial-Compartilhalgual 3.0 Não Adaptada. Ao serem publicados por esta Revista, os artigos são de livre uso em ambientes educacionais, de pesquisa e não comerciais, com atribuição de autoria obrigatória. Mais informações em http://ojs.c3sl.ufpr.br/ojs2/index.php/atoz/about/submissions\#copyrightNotice.
\end{abstract}

\begin{abstract}
Resumo
Introdução: O conceito de Comunidades de Prática (CoPs) foi introduzido no início da década de 1990 e tem sido muito popular em várias organizações, as quais reconhecem que a partilha de conhecimento é importante para o aprendizado organizacional. Essa prática mostrou ser uma abordagem estratégica e uma forma inovadora de promover a aprendizagem. O objetivo deste artigo, portanto, foi o de encontrar publicações que evidenciassem organizações que promovem o desenvolvimento das CoPs, bem como os seus aspectos relevantes no contexto organizacional.

Método: Configura-se como um estudo exploratório apoiado em Revisão Bibliográfica Sistemática (RBS) cujo processo de busca e análise ocorreu em quatro bases de dados (Portal Capes Periódicos, Scopus, Science Direct e Web of Science), utilizando-se como referência o roteiro RBS Roadmap.

Resultados: A busca retornou 43 publicações relevantes entre 1996 e 2016 (incompleto), as quais revelaram que mais de 21 empresas de diversos setores implementaram CoPs em suas organizações, desde renomadas organizações multinacionais de várias áreas, como companhias menores de prestação de serviços.

Conclusão: A pesquisa esclareceu aspectos relativos à distribuição deste tipo de produção acadêmica, a qual se concentra em publicações europeias e americanas, verificando-se o aumento do interesse dessa temática na última década. Destacam-se os benefícios das CoPs nas organizações, ressaltando-se o desenvolvimento profissional, apoio a solução de problemas, economia de tempo, sinergia entre unidades e novas estratégias competitivas.
\end{abstract}

Palavras-chave: Comunidades de prática; Organização do trabalho; Produção acadêmica

\begin{abstract}
Introduction: The concept of Communities of Practice (CoPs) was introduced in the early 1990s and it has been popular in various organizations, which recognize that the sharing of knowledge is important for organizational learning. This practice proved to be a strategic approach and an innovative way to promote learning. The purpose of this article, therefore, was to find evidence of organizations that promote the development of CoPs, as well as relevant aspects in the organizational context.

Method: It is configured as an exploratory study supported in Systematic Literature Review (RBS), whose process of search and analysis occurred in four databases (Portal Capes, Scopus, Science Direct and Web of Science), using as reference RBS script Roadmaps.

Results: The search returned 43 relevant publications between 1996 and 2016 (incomplete), which revealed that more than 21 companies from various sectors implemented CoPs in their organizations, since renowned multinational organizations in various areas such as smaller service companies. Conclusion: The research clarified aspects of the distribution of this type of academic production, which focuses on European and American publications as well as the increased interest in this theme in the last decade. We highlight the benefits of CoPs in organizations, emphasizing professional development support for problem solving, time-saving, synergy between units and new competitive strategies.
\end{abstract}

Keywords: Communities of Practice. Labor organization. Academic production.

\section{INTRODUÇÃO}

O conceito de Comunidade de Prática (CoPs) tem suas raízes na tentativa de desenvolver uma explicação de caráter social da aprendizagem humana, inspirada na antropologia e teoria social (Wenger, 2010). O termo foi cunhado por Jean Lave e Etienne Wenger em seus estudos sobre a teoria da aprendizagem, para se referir ao processo de aprendizagem. Para Wenger (2010), a aprendizagem envolve relações sociais, abrangendo neste contexto, muito mais do que a relação mestre e estudante.

A construção do conceito de CoPs se estrutura com base na aprendizagem e suas dimensões, podendo ser visto como um sistema de aprendizagem social. Os cientistas sociais têm usado versões do conceito de comunidade de prática para uma variedade de fins de análise, ainda que a origem e o uso do conceito se verifiquem na área de teoria da aprendizagem (Wenger, 2010).

As CoPs são formadas por pessoas que voluntariamente compartilham de um mesmo interesse ou paixão, interagem regularmente, trocam informações e conhecimento, buscam sustentar a comunidade e compartilham 
do aprendizado, de maneira que podem ser caracterizadas por apresentarem as seguintes dimensões: empreendimento conjunto, envolvimento mútuo e repertório compartilhado (Wenger, 2010). Terra (2005) acrescenta que os interesses comuns de aprendizado e desenvolvimento pessoal são o que mantêm as pessoas conectadas.

Ao reconhecer o conhecimento como diferencial competitivo e estratégia de negócio, as organizações passam a operacionalizar as funções da Gestão do Conhecimento o que pode ocorrer por meio de práticas que refletem as iniciativas das organizações, como o caso das Comunidades de Prática (CoPs). É comum a afirmação de que as pessoas são os recursos mais valiosos das organizações e que as relações sociais entre os trabalhadores são fontes geradoras de aprendizado, mas raramente se entende esse fato em termos de comunidades, nas quais os indivíduos criam e compartilham conhecimento. No entanto, as comunidades de prática são justamente os recursos de conhecimento mais dinâmicos e versáteis das organizações, nas quais as interações realizadas no dia a dia são tão naturais e inerentes à vivência das pessoas que, muitas vezes, nem são percebidas ou questionadas. A existência das comunidades de prática contribui para o estabelecimento das relações que possibilitam a criação e compartilhamento de conhecimento, ajudando as organizações a entender melhor o mundo, bem como possibilitando a percepção de que o aprendizado informal se dá a partir do engajamento das pessoas no fazer (Wenger, 1998). Na afirmação de Wenger (1998, p. 8), "nós prestamos atenção naquilo que esperamos ver, ouvimos aquilo que encontra espaço em nosso entendimento e agimos de acordo com nossas visões de mundo". Todas as pessoas têm formas de entender o mundo e as comunidades de prática são lugares onde é possível desenvolver, negociar e compartilhar os conhecimentos.

Acredita-se que as comunidades de prática são utilizadas nas organizações de diferentes naturezas, ramos e tamanhos e tais experiências foram publicadas em periódicos científicos. Desta forma, este estudo, em caráter exploratório, pode ser motivador para pesquisas na temática, analisando-se futuramente, por exemplo, a produção científica de determinado país.

Portanto, este artigo objetiva, por meio de uma Revisão Bibliográfica Sistemática (RBS), evidenciar organizações que promovem o desenvolvimento das CoPs, destacando-se os aspectos relevantes dessa prática no contexto organizacional.

\section{COMUNIDADES DE PRÁTICA E CONTEXTO ORGANIZACIONAL}

Os estudos desenvolvidos sobre CoPs trouxeram melhor compreensão tanto para a comunidade científica como para as empresas, pela sua importância para o sucesso das organizações (Fahey, Vasconcelos, \& Ellis, 2007; Klein, Connell, \& Meyer, 2005; Usoro, Sharratt, Tsui, \& Shekhar, 2005).

Uma CoP depende da força de três pilares: domínio, comunidade e prática (Snyder, Wenger, \& de Sousa Briggs, 2003), entendidos como:

a) Domínio: o grupo compartilha de uma paixão ou interesse em comum;

b) Comunidade: constrói-se com as relações e interações baseadas no aprendizado conjunto e o compartilhamento de informações;

c) Prática: os membros desenvolvem seu repertório próprio recorrente da prática compartilhada.

Mais do que um grupo de aprendizes, uma comunidade de prática também é uma comunidade que aprende; na visão de Pór (2002, p. 1) "não são simplesmente visionários trocando ideias em torno de águas geladas, compartilhando e beneficiando-se de outros especialistas, mas colegas compromissados em agregar as melhores práticas". As CoPs “ultrapassam os limites organizacionais" e são criadas naturalmente pela necessidade de compartilhar conhecimentos em comum (Bejarano, Pilatti, de Carvalho, \& de Oliveira, 2005). A característica principal das CoPs é a informalidade, mas isso não significa desorganização e, também, não pode ser sinônimo de networks informais ou comunidades de interesse, pois estas servem apenas para distribuir informação, enquanto que as CoPs objetivam a resolução de problemas (Wenger, McDermott, \& Snyder, 2002).

Algumas organizações assumem outras nomenclaturas para as CoPs, tais como redes de aprendizagem, grupos temáticos, clubes de tecnologia, dentre outras. As CoPs podem ser grandes ou pequenas, presenciais ou virtuais, locais ou globais, com membros internos ou externos à organização. Seu cunho é informal e intencional, porém algumas organizações as reconhecem formalmente. O interesse organizacional nas CoPs ocorre devido ao ambiente de aprendizado e a troca de conhecimento que é proporcionado (Wenger, 2002).

Wenger (2002) destaca que organizações que cultivam as Comunidades de Prática identificam ser esta, a única forma entre as estruturas organizacionais, capaz de lidar com as questões relacionadas ao conhecimento, uma vez que as CoPs:

a) Permitem aos praticantes gerenciar o conhecimento que precisam;

b) Criam uma conexão entre aprendizagem e desempenho em funções exercidas; 
c) Não se limitam, uma vez que se criam conexões além da estrutura organizacional e das restrições geográficas.

Atualmente, não existe nenhuma organização de tamanho considerável que não possua alguma forma iniciante de CoPs, uma vez que as comunidades não se limitam a gestão dos ativos de conhecimento, mas a criar valor para a organização e os membros envolvidos e de maneira abrangente (Wenger, 2002).

Terra e Gordon (2002) reforçam o exposto, apontando organizações como Xerox, World Bank, IBM, HP, Siemens, Shell, Texaco que têm promovido e apoiado formalmente as CoPs, por considerarem os benefícios que as comunidades geram em termos de inovação e conhecimento organizacional.

\section{Desenvolvimento e Suporte às Comunidades de Prática}

Cabe destacar que, uma vez estabelecida a CoP, ela precisa ser mantida adequadamente para que se sustente. Caso contrário, não trará os resultados esperados e estará fadada ao insucesso e ao seu fim.

Conforme ilustrado na Figura 1, Wenger (2002) apresenta como cultivar uma CoPs, desde a essência de sua criação, embasada na paixão pelo tema, até a apresentação dos pontos chave para a sua manutenção. Enquanto um contexto estratégico permite as comunidades encontrarem um lugar legítimo na organização, alguns elementos essenciais devem ser desenvolvidos de maneira organizada, a saber: domínio da área de interesse, relação entre membros e práticas para seu desenvolvimento. Os principais envolvidos com a comunidade de prática devem ser informados sobre o seu funcionamento, e a manutenção da CoP necessita de suporte de infraestrutura. $\mathrm{O}$ autor apresenta ainda alguns benefícios a curto e em longo prazo, com relação aos membros e à organização. Dentre estes benefícios, destacam-se o desenvolvimento profissional, ajuda com desafios, solução de problemas, economia de tempo e sinergia entre unidades e novas estratégias. Alguns desses benefícios podem ser mensurados e, desta forma, ajudam a justificar a sua implantação na organização. Além disso, Wenger (2002) também mostra elementos essenciais para o início de uma CoPs, assim como, encorajar e incentivar a participação e a integração. Destaca também, alguns fatores críticos de sucesso, com relação à comunidade e a organização.

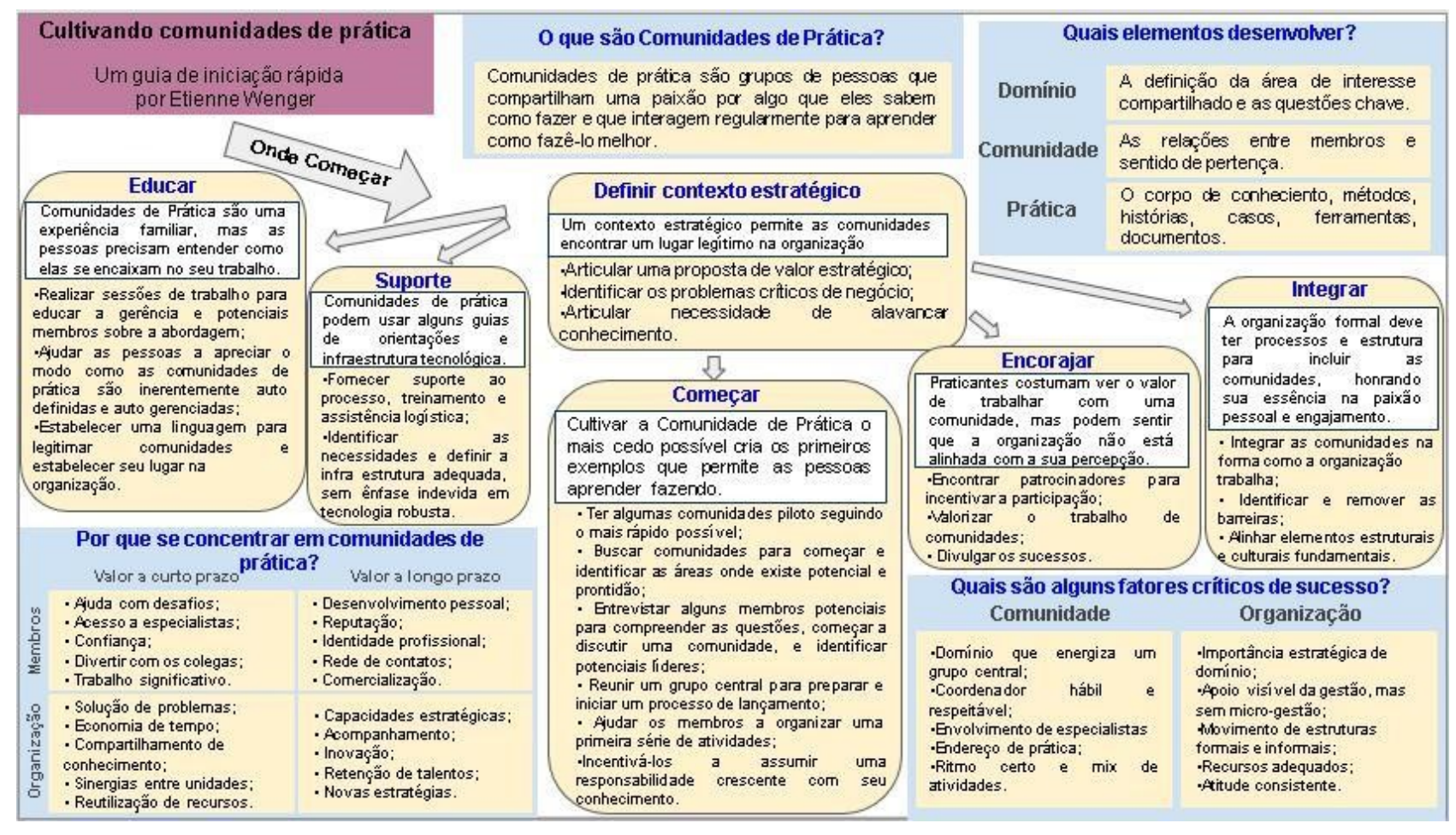

Figura 1. Cultivando Comunidades de Prática.

Fonte: Adaptado e traduzido de Wenger (2002) pelos autores (2016).

Neste processo de desenvolvimento, as Comunidades de Prática, ocupam-se de conectar pessoas, e por esse motivo devem ser considerados os níveis de participação dos seguintes membros: grupo central, participantes ativos, participantes ocasionais, participantes periféricos e participantes transacionais; assim como, a transitoriedade entre estas categorias no decorrer do envolvimento com a comunidade.

Observa-se ainda, que as comunidades evoluem continuamente, perpassando por cinco estágios de desenvolvimento: potencial, união, madura, ativa e dispersa. Esses estágios de desenvolvimento são acrescidos de sete princípios fundamentais que buscam contribuir com os objetivos organizacionais (Wenger et al., 2002):

a) desenhar a CoPs pensando na sua evolução; 
b) manter o diálogo entre a perspectiva interna e externa;

c) convidar os diferentes níveis de participação;

d) desenvolver espaços públicos e privados para a comunidade;

e) focar no valor da CoPs;

f) combinar familiaridade e estimulação;

g) criar um ritmo para a comunidade.

Os princípios apontados reforçam o caráter das CoPs, considerando que os alinhamentos das comunidades devem contribuir para alcançar os objetivos organizacionais.

\section{Benefícios de uma Comunidade de Prática}

Os estudos de Wenger (2002) apontam que o cultivo e o desenvolvimento das Comunidades de Prática agregam benefícios às organizações, em curto prazo, visando à melhoria dos resultados dos negócios e em longo prazo, tendo como objetivo o desenvolvimento de capacidades organizacionais (Quadro 1).

\begin{tabular}{|l|l|}
\hline Benefício de curto prazo & Benefício de longo prazo \\
\hline Resolução de problemas & Capacidade de executar um plano estratégico \\
Agilidade nas respostas às questões & Autoridade com o cliente \\
Redução de tempo e custo & Aumento da retenção de talentos \\
Melhora na qualidade das decisões & Capacidade para projetos de desenvolvimento do conhecimento \\
Maior perspectiva sobre os problemas & Fórum para benchmarking com as unidades da organização \\
Sinergia entre as unidades & Alianças baseadas no conhecimento \\
Recursos para implementações de estratégias & Surgimento de capacidades não planejadas \\
Qualidade de garantia reforçada & Capacidade de desenvolver novas opções estratégicas \\
Capacidade de assumir riscos com o apoio da comunidade & Capacidade de prever os desenvolvimentos tecnológicos \\
& Capacidade de aproveitar as oportunidades de mercado emergen- \\
\hline
\end{tabular}

Quadro 1. Benefício das CoPs às Organizações - Curto e Longo Prazo.

Fonte: Adaptado e traduzido de Wenger et al. (2002) pelos autores (2016).

As CoPs, todavia, só obtêm os resultados e benefícios apontados se houver a participação efetiva das pessoas. Os benefícios aos membros no âmbito do trabalho podem ser o de contribuir com os desafios, acessar o conhecimento necessário, melhorar a capacidade de contribuição com equipes de trabalho, confiança na e para resolução de problemas, maior envolvimento com colegas de trabalho, participação significativa e senso de pertencimento. As CoPs contribuem ainda para o desenvolvimento profissional, com a disponibilização de espaços para o compartilhamento de conhecimentos, desenvolvimento de redes de relacionamento pessoais em longo prazo, aprendizado com especialistas, resolução de problemas complexos e desenvolvimento de perspectivas mais amplas da organização (Wenger et al., 2002).

As CoPs estão inseridas e difundidas no contexto organizacional, apesar da característica informal que as constitui. Entretanto, pretende-se aqui investigar os estudos que relatem essa prática e verificar como as CoPs são promovidas e aplicadas (intencionalmente em seus contextos, ou não) nas organizações.

Apresentam-se a seguir os procedimentos metodológicos para a realização desta investigação em que se emprega um conjunto de passos, técnicas e ferramentas com o intuito de realizar um estudo sobre a aplicação das Comunidades de Práticas no contexto organizacional.

\section{METODOLOGIA}

Esta pesquisa caracteriza-se como exploratória com base na Revisão Bibliográfica Sistemática (RBS) ou Revisão Sistemática da Literatura (RSL), ou seja, é uma forma de identificar, avaliar e interpretar as pesquisas existentes, relevantes a uma questão de pesquisa, tópico ou fenômeno de interesse (Kitchenham, 2004). Este tipo de técnica permite identificar as contribuições chave para uma área ou para uma questão. Além disso, oferece um procedimento estatístico a fim de sintetizar conclusões, a partir das quais é possível conseguir uma confiabilidade indisponível em estudos individualizados (Tranfield, Denyer, \& Smart, 2003).

O método de pesquisa utilizado de revisão bibliográfica sistemática (RBS) foi adaptado do roteiro proposto por Conforto, Amaral, e da Silva (2011), utilizando-se como referência o RBS Roadmap (Figura 2).

A pesquisa foi conduzida por um conjunto de etapas, ordenadamente dispostas, visando à realização da investigação proposta, com o intuito de trabalhar com uma grande quantidade de dados e expor o cenário do assunto investigado, com a obtenção de informações confiáveis acerca de comunidades de prática em organizações. 


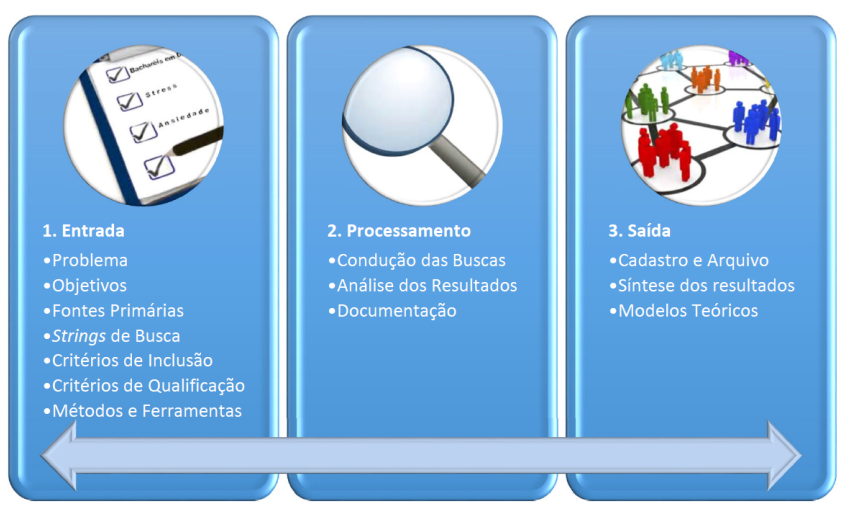

Figura 2. Modelo Adaptado do Roteiro de Conforto et al. (2011) para Condução da Revisão Bibliográfica Sistemática - RBS Roadmap. Fonte: Elaborada pelos autores (2016).

\section{Condução da Revisão Bibliográfica Sistemática}

A problemática da pesquisa norteou a condução desta RBS ao explicitar respostas à seguinte questão, "quais empresas estão inserindo as Comunidades de Prática no seu contexto organizacional?”. Tendo em vista que as organizações buscam a implantação de novas estruturas que possibilitem aperfeiçoar a sua gestão do conhecimento, esse processo destaca-se como uma maneira de promover essa busca.

A construção da estratégia de busca (strings) seguiu um processo de seleção, testes e ajustes, por meio de testes de combinação de palavras, termos, e de utilização de operadores lógicos de busca booleana. A partir de uma revisão bibliográfica preliminar, sem o rigor de uma revisão sistemática, foram identificadas as palavras-chave que caracterizam o tema investigado, sendo "community of practice" o principal termo de busca, acompanhado de "organizations", "companies", "enterprises" e "corporations". Adotou-se ainda, o termo "communities of practice" para a ampliação do escopo. As palavras-chave de busca foram utilizadas na língua inglesa pelo fato de que as publicações, mesmo de outra língua, são indexadas por palavras-chave e resumos na língua inglesa. Acredita-se que esse critério possibilitou a ampliação da abrangência da pesquisa sobre comunidades de prática.

Já os critérios de inclusão das publicações consideraram o objetivo da pesquisa (buscar estudos que demonstrassem a aplicação da prática organizacional de comunidade de prática em um ambiente corporativo) com vistas a evidenciar organizações que promovem as CoPs. Como critério essencial de qualificação elegeu-se o método de pesquisa adotado pelos artigos como sendo estudos de caso. Ressalta-se que a investigação se restringiu a estudos publicados nos últimos 20 anos, ou seja, compreendendo o período de 1996 a 2016 (incompleto). Foram rastreados, primeiramente, os títulos, os resumos e palavras-chave das publicações nas bases de dados do Science Direct e Scopus, e do tópico nos Periódicos Capes e na Web of Science. Posteriormente, os artigos selecionados foram analisados na íntegra.

As palavras-chave adotadas foram empregadas na busca de registros em quatro bases de dados: Portal de Periódico Capes, Scopus, Science Direct e Web of Science. Todas se constituem plataformas online, as quais permitem acesso a publicações científicas de diversos países nas principais áreas do conhecimento (Tabela 1).

\begin{tabular}{|c|c|c|c|c|c|c|}
\hline \multirow{2}{*}{\multicolumn{2}{|c|}{ Especificações }} & \multicolumn{4}{|c|}{ Base de Dados } & \multirow[t]{2}{*}{ Total } \\
\hline & & Periódicos Capes & Scopus & Science Direct & Web of Science & \\
\hline \multirow{4}{*}{ Palavras-Chave } & Community of practice and organizations & 198 & 1406 & 128 & 190 & 1922 \\
\hline & Community of practice and companies & 29 & 340 & 23 & 45 & 437 \\
\hline & Community of practice and enterprises & 21 & 339 & 20 & 14 & 394 \\
\hline & Community of practice and corporations & 11 & 67 & 10 & 9 & 97 \\
\hline \multicolumn{2}{|c|}{ Total de artigos encontrados (sem filtro) } & 259 & 2152 & 181 & 258 & 2850 \\
\hline \multirow{4}{*}{ Filtros } & $\begin{array}{l}1^{\circ} \text { Filtro - (Leitura do título, resumo e } \\
\text { palavras-chave - menos } 2.616 \text { artigos) }\end{array}$ & 35 & 162 & 15 & 22 & 234 \\
\hline & $\begin{array}{l}2^{\circ} \text { Filtro - (Exclusão de duplicidades - me- } \\
\text { nos } 151 \text { artigos) }\end{array}$ & 11 & 55 & 5 & 12 & 83 \\
\hline & $\begin{array}{l}3^{\circ} \text { Filtro - (Leitura da introdução e conclu- } \\
\text { são - menos } 22 \text { artigos) }\end{array}$ & 13 & 41 & 4 & 3 & 61 \\
\hline & $\begin{array}{l}3^{\circ} \text { Filtro - (Leitura completa - menos } 18 \\
\text { artigos) }\end{array}$ & 13 & 25 & 2 & 3 & 43 \\
\hline \multicolumn{2}{|c|}{ Total de pesquisas relevantes } & 13 & 25 & 2 & 3 & 43 \\
\hline
\end{tabular}

Tabela 1. Resultados da pesquisa nas Bases de Dados conforme as palavras-chave.

Fonte: Elaborada pelos autores (2016).

Preliminarmente, com o uso das palavras-chave selecionadas, foram obtidos 2.850 trabalhos, distribuídos nas bases de dados. Destaca-se a quantidade de publicações encontradas na base de dados Scopus em relação às demais, correspondendo a aproximadamente $76 \%$ do total. 
Para a aplicação do primeiro filtro, realizou-se a leitura dos títulos, resumos e palavras-chave. As publicações que estavam alinhadas com o objetivo da pesquisa foram selecionadas para o próximo filtro e o restante, 2.616 trabalhos, foram considerados irrelevantes para esta revisão. Ressalta-se que em muitos trabalhos o $1^{\circ}$ filtro não foi suficiente para constatar se a publicação atendia aos objetivos e critérios de inclusão, uma vez que existiam resumos concisos que dificultavam a compreensão da fundamentação do trabalho. Ante o exposto, optou-se em mantê-los na lista de artigos e submetê-los ao $3^{\circ}$ Filtro.

Em virtude do uso de quatro bases de dados, havia a possibilidade de pesquisas estarem indexadas em mais de uma base de dados, além de existir a repetição devido a utilização de várias palavras-chave. Desta maneira, as duplicidades foram verificadas excluindo-se outros 151 trabalhos. Na sequência, para os 83 artigos remanescentes recorreu-se ao $3^{\circ}$ filtro (leitura da introdução e da conclusão dos trabalhos), o que resultou em 61 artigos.

Para a análise de conteúdo dos artigos, aplicou-se um $4^{\circ}$ filtro (leitura completa dos artigos), sendo excluídos 18 artigos, por não serem de acesso livre. Diante disso, foram considerados relevantes 43 artigos para análise das organizações em que as CoPs foram estudadas.

Para análise desses 43 trabalhos estabeleceu-se um roteiro incluindo: a temporalidade das publicações, as organizações e setores onde foram implementadas as CoPs, as metodologias, processos e ferramentas de TI para as CoPs; o enfoque do estudo e apontamentos sobre a utilização das CoPs como vantagem competitiva, os motivos de sucesso e fracasso de suas implementações e os benefícios identificados pelas organizações quanto a sua contribuição institucional. Os trabalhos foram categorizados em três grupos de artigos. No primeiro grupo, foram incluídos artigos que evidenciam a utilização de espaços compartilhados. No segundo grupo, foram incluídos artigos que destacam projeto e modelo customizado desenvolvido especialmente para a organização. No último grupo, foram incluídos artigos que destacam o nome e setor de aplicação das CoPs.

\section{RESULTADOS}

Ao se considerarem as 43 publicações selecionadas pela RBS para a pesquisa foram possíveis analisar alguns resultados.

A Tabela 1 indica que as pesquisas aderentes estão predominantemente indexadas na base Scopus. Ainda neste estudo foi possível averiguar as regiões onde as empresas foram pesquisadas, apesar de que apenas 31 publicações apresentavam o local das pesquisas, conforme Figura 3.

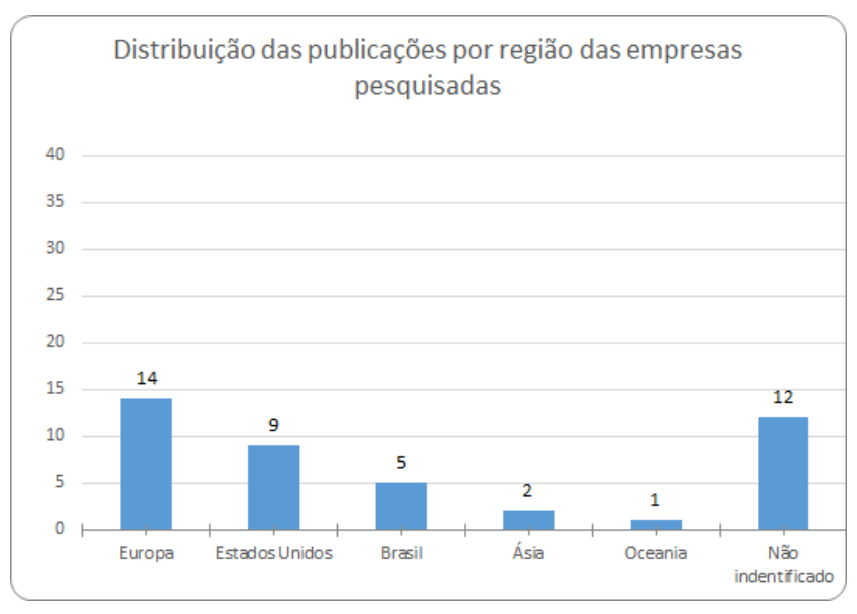

Figura 3. Distribuição das publicações por região das empresas pesquisadas. Fonte: Elaborada pelos autores (2016).

Na Figura 3, evidencia-se que, majoritariamente, as pesquisas foram realizadas na Europa e, em seguida, nos Estados Unidos, enquanto que Brasil, Ásia e Oceania apresentam menos publicações relacionadas ao tema. Além disso, considerando os trabalhos relevantes, procedeu-se uma análise de distribuição temporal das pesquisas, com o propósito de evidenciar o desenvolvimento da temática alvo deste artigo. O resultado pode ser visualizado na Figura 4.

A Figura 4 permite observar que existe um aumento na quantidade de artigos correlatos produzidos em 2008, vindo a decair em 2009 e 2010. Porém, em 2011 tem-se o auge das publicações envolvendo CoPs em organizações, de acordo com os filtros e critérios de seleção. Poucos artigos com este critério foram publicados de 1996 a 2000, ficando a maioria para o último decênio. 


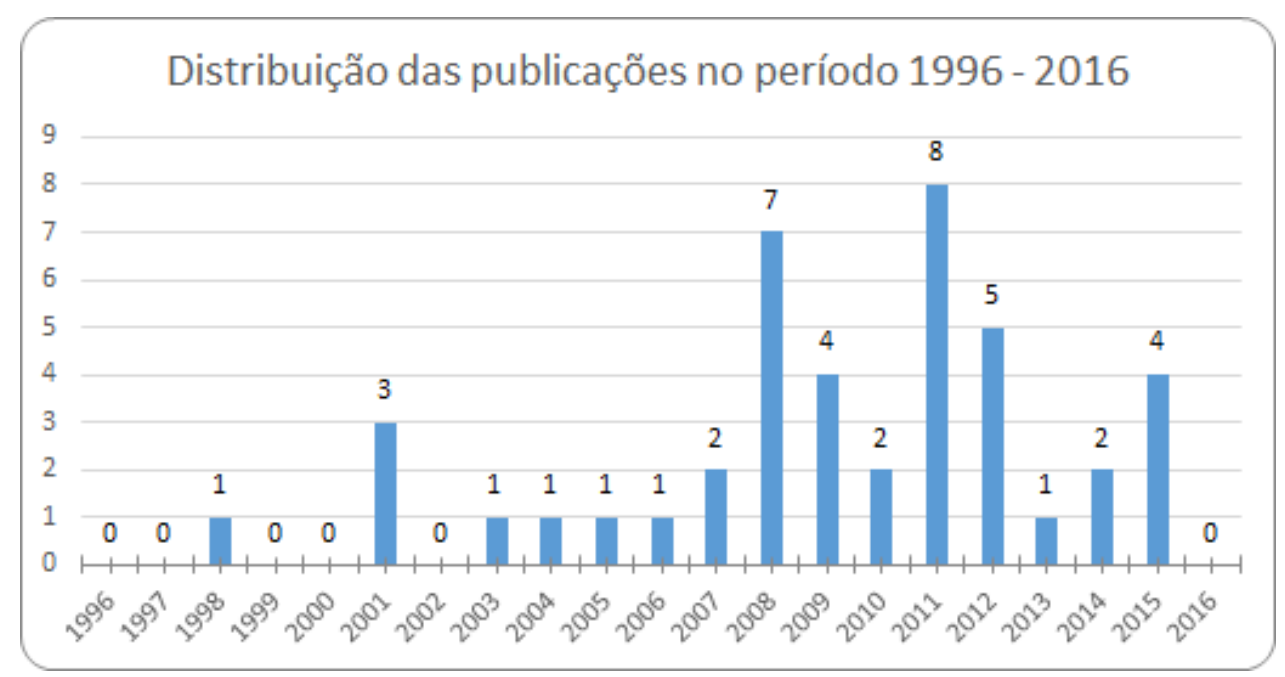

Figura 4. Distribuição das Publicações no Período 1996 - 2016.

Fonte: Elaborada pelos autores (2016).

\section{Artigos que evidenciam espaços compartilhados para CoPs}

Foram identificados 17 artigos neste grupo, nos quais se evidencia a utilização dos espaços compartilhados na web dedicados exclusivamente aos membros das CoPs. Na maioria dos casos, isso implicou em uma mudança de cultura dos colaboradores, uma vez que se almejava explorar o uso da tecnologia da informação para apoiar a partilha de conhecimentos dentro, e até mesmo entre outras CoPs. Entretanto, em uma das pesquisas, destacase que é possível que uma CoP virtual seja preterida pelos colaboradores (mesmo em uma empresa de tecnologia da informação) não podendo assim ter seu valor superestimado, devendo-se valorizar concomitantemente a qualidade do diálogo entre os participantes.

Constatou-se que o conhecimento das CoPs era aprimorado e partilhado entre todos, possibilitando a remoção de barreiras organizacionais e o aumento da confiança dos membros, desempenhando um papel importante na aprovação de novos produtos, procedimentos e melhorias de processos.

\section{Artigos que evidenciam Projeto e Modelo Customizados}

Foram analisados 21 artigos, sendo que onze relatam o desenho de um modelo próprio aplicado na organização e de possível replicação. Destaca-se o apontamento em um artigo para a criação de um modelo de governança hierárquica para a CoP e, em um segundo artigo, o estabelecido do uso da gestão enxuta na CoP. Em ambos os casos foram identificados a resistência ao modelo, a baixa interação entre os membros e a falta de identificação com a CoP.

Verificou-se a indicação de CoPs virtuais e suporte tecnológico, porém sem menção a softwares ou programas utilizados no processo. Dois artigos mencionaram ferramentas como parte importante nas CoPs, sendo uma delas, o design de interface do usuário, utilizada para o contexto de mudança organizacional e o modelo de detecção de ponto de troca com inferências estatísticas para avaliar a probabilidade de alteração na taxa de adoção de procedimentos operacionais novos e revisados, após a implementação das CoPs. Oito artigos destacaram os processos que envolvem a participação, o desenvolvimento, benefícios identificados e o sucesso e fracasso das CoPs. O incentivo a partilha do conhecimento, o alinhamento a estratégia organizacional e a liderança exercida nas CoPs são principais itens relatados para o sucesso das comunidades.

\section{Artigos que evidenciam o nome da organização e/ou setor de aplicação da CoP}

Identificou-se ainda, que o desenvolvimento das CoPs tem sido promovido por organizações que reconhecem a contribuição que as comunidades podem ter para a criação e compartilhamento de conhecimento. Algumas publicações continham os nomes das organizações, enquanto outras apenas mencionavam os setores de atuação dessas. Assim, destaca-se, no Quadro 2, as organizações identificadas e no Quadro 3 setores, ambos que tiveram a experiência de CoPs publicados, e selecionados dentro da RBS, sendo estas de diferentes setores, assim como grandes organizações e multinacionais, ou ainda pequenas organizações, como por exemplo a comunidade online de viagem (Quadro 3).

Diante desse panorama, constata-se que as organizações que buscaram implementar as comunidades de prática precisaram criar um ambiente propício para que elas pudessem se estabelecer e desenvolver. Ainda, para atingirem um grau satisfatório, foi necessário a integração à organização e apoiadas de maneira incondicional 


\begin{tabular}{|l|l|}
\hline \multicolumn{2}{|c|}{ Organizações identificadas } \\
\hline Aeronautics and Space Administration (NASA) & National Computer Systems Pte Ltda. \\
Bearing Point & Nokia Corporation \\
Buckman Labs & Oracle \\
Daimler Chrysler & Price Waterhouse Coopers \\
Departamento de Justiça Catalã e Agência Catalã de Proteção à Saúde & Robert Bosch GmbH (Bosch) \\
Elektro Eletricidade S.A & Rolls-Royce \\
HP & Schneider Electric \\
Holcim & Siemens \\
IBM & Valença \& Associados Aprendizagem Organizacional \\
Inter-American Development Bank & Xerox \\
\hline
\end{tabular}

Quadro 2. Organizações que promovem Comunidades de Prática com base nos artigos analisados. Fonte: Elaborado pelos autores com base na análise das publicações (2016).

\begin{tabular}{|l|l|}
\hline \multicolumn{2}{|c|}{ Setores identificados } \\
\hline Banco Federal & Logística \\
Biblioteca digital & Multinacional de mineração e processamento de minério \\
Comunidade online Chinesa de viagem & Multinacional de petróleo \\
Construção e engenharia & Multinacional de T.I. \\
Designers de interface de usuários & Setor energético \\
Divisão química de uma multinacional & Subsidiária norueguesa automobilística \\
Empresa escandinava de gerenciamento de projetos & Turismo \\
Indústria automotiva & Indústria química especializada \\
\hline
\end{tabular}

Quadro 3. Setores que promovem Comunidades de Prática com base nos artigos analisados. Fonte: Elaborado pelos autores com base na análise das publicações (2016).

pelos gestores, os quais estiveram prontos para intervir quando surgiram obstáculos que podiam impedir a evolução das comunidades.

Os resultados apontam que a literatura sobre a aplicação de Comunidades de prática nas organizações (61 artigos) é escassa em relação ao total de artigos (2.850) encontrados sobre o tema. Constatou-se, ainda, que os artigos analisados relatam metodologias, processos e ferramentas de tecnologia da informação para o suporte as CoPs, dentre outros.

\section{CONSIDERAÇÕES FINAIS}

Este trabalho apresentou o conceito de Comunidades de Prática, suas contribuições organizacionais e seu desenvolvimento e suporte seguido de uma seleção dos trabalhos empíricos, nas bases de dados Portal de Periódico Capes, Scopus, Science Direct e Web of Science, sobre Comunidades de Prática e a inserção dessas no contexto organizacional, evidenciando organizações que às promovem.

As informações levantadas e analisadas possibilitaram uma compreensão geral quanto às produções acadêmicas sobre CoPs e a sua inserção no contexto organizacional nos últimos 20 anos (período de 1996 a 2016 incompleto).

Majoritariamente, as pesquisas foram realizadas na Europa, seguido pelos Estados Unidos, sendo a maioria das publicações do último decênio. Ainda foi possível constatar que, até o momento, não são muitos os estudos que relatam as aplicações das CoPs nas organizações. Observa-se que a implementação da Comunidade de Prática e o engajamento dos membros com o compartilhamento e aprendizagem baseada em interesses comuns, podem melhorar o desempenho organizacional. Além de gerar com isso, um impacto sobre a resolução de problemas específicos e vantagem competitiva organizacional.

Devido à utilização do procedimento metodológico baseado na revisão sistemática bibliográfica, este trabalho possibilita o desenvolvimento de outras pesquisas e temas não abordados no presente artigo. Neste sentido, sugerem-se futuras pesquisas para verificar os resultados encontrados nas publicações selecionadas, contrapondo-as às publicações de Etienne Wenger a fim de identificar aspectos relevantes e características intrínsecas às aplicações organizacionais.

Assim sendo, as Comunidades de Prática permitem várias pesquisas quanto as suas conexões, formas de trabalho e convivência de maneira conjunta e natural nas organizações de diferentes segmentos profissionais. 


\section{REFERÊNCIAS}

Bejarano, V. C., Pilatti, L. A., de Carvalho, H. G., \& de Oliveira, A. C. (2005). Equipes e comunidades de prática como estruturas complementares na gestão do conhecimento organizacional. In $X X V$ Encontro Nacional de Engenharia de Produção. Porto Alegre, RS, Brasil.

Conforto, E. C., Amaral, D. C., \& da Silva, S. L. (2011). Roteiro para revisão bibliográfica sistemática: aplicação no desenvolvimento de produtos e gerenciamento de projetos. In VIII Congresso Brasileiro de Gestão de Desenvolvimento de Produto. Porto Alegre, RS, Brasil: UFRS. Recuperado de http://www.ufrgs.br/cbgdp2011/downloads/9149.pdf

Fahey, R., Vasconcelos, A. C., \& Ellis, D. (2007, Aug.). The impact of rewards within communities of practice: a study of the SAP online global community. Knowledge Management Research \& Practice, 5(3), 186-198. doi: 10.1057/palgrave.kmrp. 8500140

Kitchenham, B. (2004, Jul.). Procedures for performing systematic reviews. Recuperado de http://people.ucalgary.ca/ $\sim$ medlibr/kitchenham_2004.pdf (Keele University Technical Report TR/SE-0401)

Klein, J. H., Connell, N. A. D., \& Meyer, E. (2005). Knowledge characteristics of communities of practice. Knowledge Management Research \& Practice, 3(2), 106-114. doi: 10.1057/palgrave.kmrp. 8500055

Pór, G. (2002). What is a community of practice? Recuperado em 2 dez. 2015, de http://www.co-i-I.com/coil/knowledge -garden/cop/definitions.shtml

Snyder, W. M., Wenger, E., \& de Sousa Briggs, X. (2003). Communities of practice in government: Leveraging knowledge for performance. The Public Manager, 32(4), 17-21. Recuperado de http://www.civicstewardship.com/uploads/ 03.12.26_Snyder_et_al._Public_Manager_Winter_2003-2004 _v32_n4.pdf

Terra, J. C. C. (2005). Gestão do conhecimento: O grande desafio empresarial. São Paulo, SP, Brasil: Elsevier.

Terra, J. C. C., \& Gordon, C. (2002). Portais corporativos: a revolução na gestão do conhecimento. São Paulo, SP, Brasil: Elsevier.

Tranfield, D., Denyer, D., \& Smart, P. (2003, Sept.). Towards a methodology for developing evidence-informed management knowledge by means of systematic review. British Journal of Management, 14(3), 207-222. doi: 10.1111/14678551.00375

Usoro, A., Sharratt, M. W., Tsui, E., \& Shekhar, S. (2005, Sept.). Trust as an antecedent to knowledge sharing in virtual communities of practice. Knowledge Management Research \& Practice, 5(3), 199-2012. doi: 10.1057/palgrave.kmrp.8500143

Wenger, E. (1998). Communities of practice: learning, meaning and identity. Cambridge, UK: Cambridge University.

Wenger, E. (2002). Cultivating communities of practice: a quick start-up guide for communities of practice. Recuperado de http://wenger-trayner.com/project/community-of -practice-start-up-guide/

Wenger, E. (2010). Communities of practice and social learning systems: the career of a concept. In C. Blackmore (Ed.), Social learning systems and communities of practice (p. 179-198). London, UK: Springer. doi: 10.1007/978-1-84996133-2_11

Wenger, E., McDermott, R. A., \& Snyder, W. (2002). Cultiva- ting communities of practice: a guide to managing knowledge. Boston, MA, USA: Harvard Business School.
Como citar este artigo (APA):

Fernandes, F. R., Cardoso, T. A., Capaverde, L. Z. \& Silva, H. F. N. (2016). Comunidades de prática: uma revisão bibliográfica sistemática sobre casos de aplicação organizacional. AtoZ: novas práticas em informação e conhecimento, 5(1), 44 - 52. Recuperado de: http://dx.doi.org/10.5380/atoz.v5i1.46691 